\title{
Las casas de Augusta Emerita: urbanismo y modelos de arquitectura residencial
}

\section{Roman houses in Augusta Emerita: town-planning and residential architecture models}

\author{
Álvaro Corrales Álvarez \\ Universidad Pablo de Olavide de Sevilla. Departamento de Geografía, Historia y Filosofía \\ Carretera de Utrera, km 1, E-41013 Sevilla \\ acoralv@upo.es
}

L'objectiu principal d'aquesta investigació és una reflexió nova sobre la transmissió dels models arquitectònics romans a les construccions privades emeritenques, a través del filtre d'una tipologia de cases procedent de la tradició mediterrània. La fundació de la colònia Augusta Emerita al segle I a. C. suposa l'assimilació de les tipologies constructives de residències al voltant d'atris i peristils, models d'èxit, testats a tota la geografia de l'Imperi. Des d'un punt de vista arquitectònic, les cases emeritenques no pertanyen només a la tradició itàlica, sinó que presenten elements de caracterització diversa.

\section{PARAULES CLAU}

EDILICIA RESIDENCIAL, URBANÍSTICA, SIG, LUSITANIA, S. I A. C. - IV D. C.

This paper proposes to undertake a new reflection on Roman architectural models and their transmission in the case of the private houses of Augusta Emerita (Mérida, Spain), through the filter of a range of house types of Mediterranean tradition. The foundation of the colonia Augusta Emerita in the first century $\mathrm{BC}$ led to the assimilation of the construction typologies of residences focused on atria and peristyles, successful models tested across the Empire. From an architectural point of view, the houses of Augusta Emerita do not belong only to the Italian tradition, but present elements of different characterization.

\section{KEYWORDS}

HOUSES, URBAN LAYOUT, GIS, LUSITANIA, IST CENTURY BC-4TH CENTURY AD

El objetivo principal de esta investigación es una nueva reflexión sobre la transmisión de los modelos arquitectónicos romanos en las construcciones privadas emeritenses, a través del filtro de una tipología de casas procedente de la tradición mediterránea. La fundación de la colonia Augusta Emerita en el siglo i a. C. supone la asimilación de las tipologías constructivas de residencias en torno a atrios y peristilos, modelos de éxito, testados en toda la geografía del Imperio. Desde un punto de vista arquitectónico, las casas emeritenses no pertenecen solo a la tradición itálica, sino que presentan elementos de diversa caracterización. 


\section{Introducción}

La fundación ex novo de la colonia Augusta Emerita en el año 25 a. C. representa uno de los fenómenos urbanos de referencia de la provincia Lusitania. Entre el año 16 y el 13 a. C., el emperador Augusto incrementó el impacto de la ciudad en la gestión del territorio con la concesión del rango de capital provincial. Este nuevo status fue aceptado por las poblaciones locales, como testimonia el acuerdo de hospitalidad con los Martienses en torno al 6 d. C. (Saquete, 2005: 177). La creación de la colonia responde a un programa general de fundaciones en relación con la propaganda augustea, fruto de una planificación global destinada a establecer nuevas relaciones entre el poder central y local (Mateos, 2011: 128). El emperador y sus legados desempeñaron un papel fundamental en el nuevo proyecto, con la construcción de infraestructuras públicas para el funcionamiento urbano y la administración de las poblaciones circundantes, que comenzaron un proceso de integración cultural favorecido por una gestión central del territorio y de los recursos minerales.

El análisis de la tipología de hábitat cívico contribuye al conocimiento de los grupos sociales, así como de las formas de la familia y sus condiciones de vida. El estudio de los edificios residenciales hace posible la identificación de las particularidades, las semejanzas y diferencias en los procesos constructivos de las casas. Este aspecto resulta necesario para poder confrontar la evolución de las dinámicas de ocupación urbanas en la geografía del mundo romano. A pesar de las tipologías consolidadas de organización espacial en torno a atrios y peristilos, se hace necesario someter a todos los ambientes domésticos a un análisis específico para examinar las fórmulas de circulación de los diferentes proyectos arquitectónicos de manera homogénea. En este sentido, el camino a seguir debería ser el de aumentar una serie de estudios de contextos de vivienda a nivel regional (Gros, 2001: 137) para centrarse en la transmisión de los diversos modelos arquitectónicos en relación con las tradiciones locales, el conocimiento técnico de los trabajadores y la adaptación del modelo a las condiciones ambientales y topográficas de las distintas ciudades y territorios.

La aportación que se plantea se basa fundamentalmente en tres aspectos: una propuesta metodológica para la identificación de ambientes y comparación de modelos arquitectónicos residenciales en yacimientos urbanos; el examen de las relaciones entre urbanística y los modelos de la arquitectura doméstica, y, finalmente, una breve relación entre del origen de los arquetipos de vestíbulos y salones triclinares en la edilicia doméstica emeritense. Así, la coexistencia de modelos muy diferentes o, por el contrario, la presencia de esquemas similares de diferentes períodos facilitan el estudio de la relación entre los elementos formales y la circulación y transmisión de modelos. La base del estudio de las casas romanas de Mérida es el catálogo de 178 viviendas, divididas en nueve regiones, elaborado recientemente (Corrales, 2016: 168) y actualizado con la información de las últimas viviendas documentadas en la colonia, hasta sumar un total de 221 (fig. 1), aunque por cuestiones de espacio, trataremos en adelante solo de la edilicia residencial más representativa. 


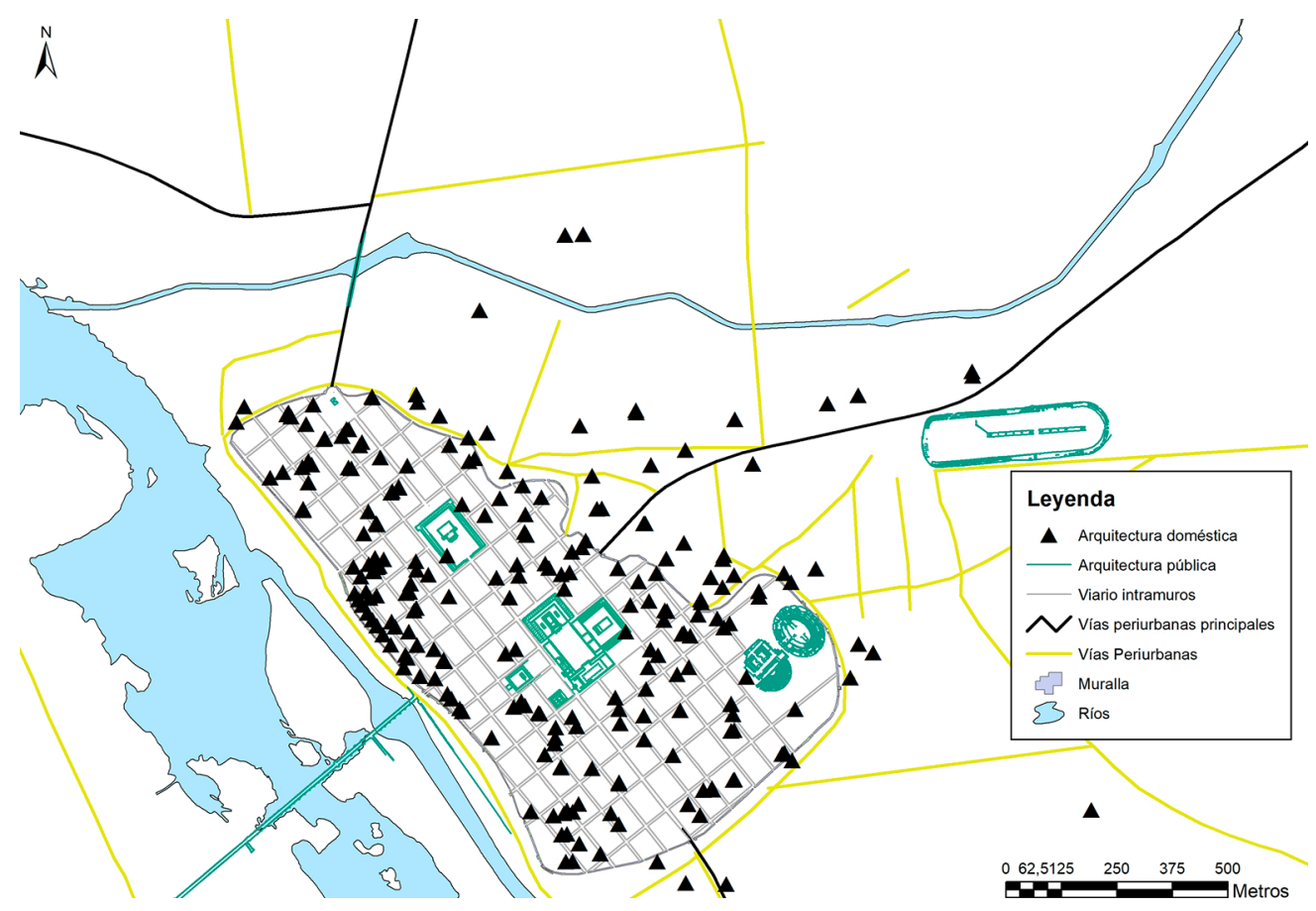

Figura 1. Mapa que muestra la arquitectura doméstica de Augusta Emerita fuente: elaboración del autor a partir de planimetrías del Consorcio de Mérida).

\section{Propuesta metodológica para la identificación y comparación de modelos arquitectónicos residenciales en yacimientos urbanos}

La línea metodológica propuesta en esta investigación para la caracterización y cotejo de las tipologías de viviendas urbanas, tal y como propuso Ellis (1999), pasa por el reconocimiento del espacio de circulación, aunado a las consideraciones sobre elementos arquitectónicos ya empleados por Rebuffat $(1969,1974)$ y tomados de nuevo por Bonini $(2006)$. En este sentido, el reconocimiento de áreas funcionales y las relaciones de los ambientes de la casa pueden servirnos como enfoque para la definición del modelo residencial a partir del ingreso, los espacios de distribución y la sala social.

En el plano historiográfico, la investigación especializada razonó que el diseño de las viviendas hundía sus raíces en la segregación de ambientes públicos y privados y en la génesis de modelos de circulación para propietarios, invitados y siervos (Hillier y Hanson, 1984; Wallace-Hadrill, 1988: 43-97). Las mayores limitaciones a estas hipótesis son los requisitos iniciales de las propuestas. Así, para poder aplicar fórmulas geométricas 
se debe conocer la planta completa de la vivienda, hecho que rara vez sucede en una ciudad superpuesta como Mérida. De hecho, el panorama más frecuente es contar con plantas parciales en las que, a menudo, la fragmentación de las estructuras no permite conocer con suficiente nivel de detalle los restos como para proponer la funcionalidad de las estancias.

Actualmente existen dos tipos de aproximaciones para establecer la funcionalidad de los espacios en la casa. La primera se basa en los presupuestos de la household archeology (arqueología doméstica), que presta una especial atención al análisis de la cultura material recuperada del registro arqueológico. Este enfoque ha dado buenos resultados en Pompeya (Allison, 2004) y también en la península ibérica (Bermejo, 2014). La dificultad para esta propuesta radica en la premisa inicial: el análisis de contextos materiales estratigráficamente inalterados. En la arqueología de las ciudades superpuestas resulta un recurso muy poco frecuente. Otro hecho que suele agravar este problema es la falta de publicación de resultados de excavaciones.

El otro acercamiento se debe al examen de las fuentes literarias antiguas que proporcionan descripciones sobre determinados estándares de habitaciones. Esta documentación aporta datos sobre dimensiones o decoración que resultan de utilidad para la identificación de espacios. Sin embargo, esta aproximación también posee algunas limitaciones (Allison, 1992: 235-249). Asimismo, algunas de las estancias no estaban diseñadas para una única función, sino que se corresponden con espacios polivalentes (Riggsby, 1997: 36-56) cuyo uso podía variar a lo largo de las franjas horarias del día o a lo largo de las estaciones (Nevett, 1997: 295). En última instancia, parece razonable pensar que unos espacios estaban destinados al uso y disfrute de los propietarios y otros estaban vinculados al trabajo y obligación de los siervos (Ellis, 1999: 75-98). Habitualmente, las habitaciones del dominus y su familia son espacios difíciles de alcanzar, pues parecen cerrados a la circulación y suelen poseer una rica decoración; por otra parte, las dependencias serviles se tienden a identificar por la ausencia de estos marcadores (Peñalver, 2019: 105-131).

El problema de los yacimientos urbanos es que a menudo no permiten conocer con suficiente nivel de detalle ni siquiera los ingresos, espacios de circulación y salas sociales que resultan esenciales para conocer la planta de una casa. El conjunto de herramientas proporcionadas por los Sistemas de Información Geográfica (en adelante SIG) sirven, en esta investigación, para coordinar una ingente cantidad de informaciones heterogéneas en un único entorno topográfico cerrado. La principal ventaja de los SIG utilizada durante este trabajo es que permitió apreciar la continuación de estructuras pertenecientes a diferentes excavaciones pero que mantenían una misma orientación.

En la calle Viñeros, se excavaron dos solares en los números 7 y 12, inmuebles afrontados en lados diferentes, mediante intervenciones llevadas a cabo en años diversos (Palma, 2005: 247-260; Chamizo, 2006: 243-260). Al insertar en el SIG las estructuras documentadas en relación con la trama urbana antigua, se reveló que los restos se encontraban al interior de una misma manzana residencial, pues el trazado de la calle Viñeros no existía en época romana. El análisis de las técnicas edilicias y la cultura material permitió establecer 


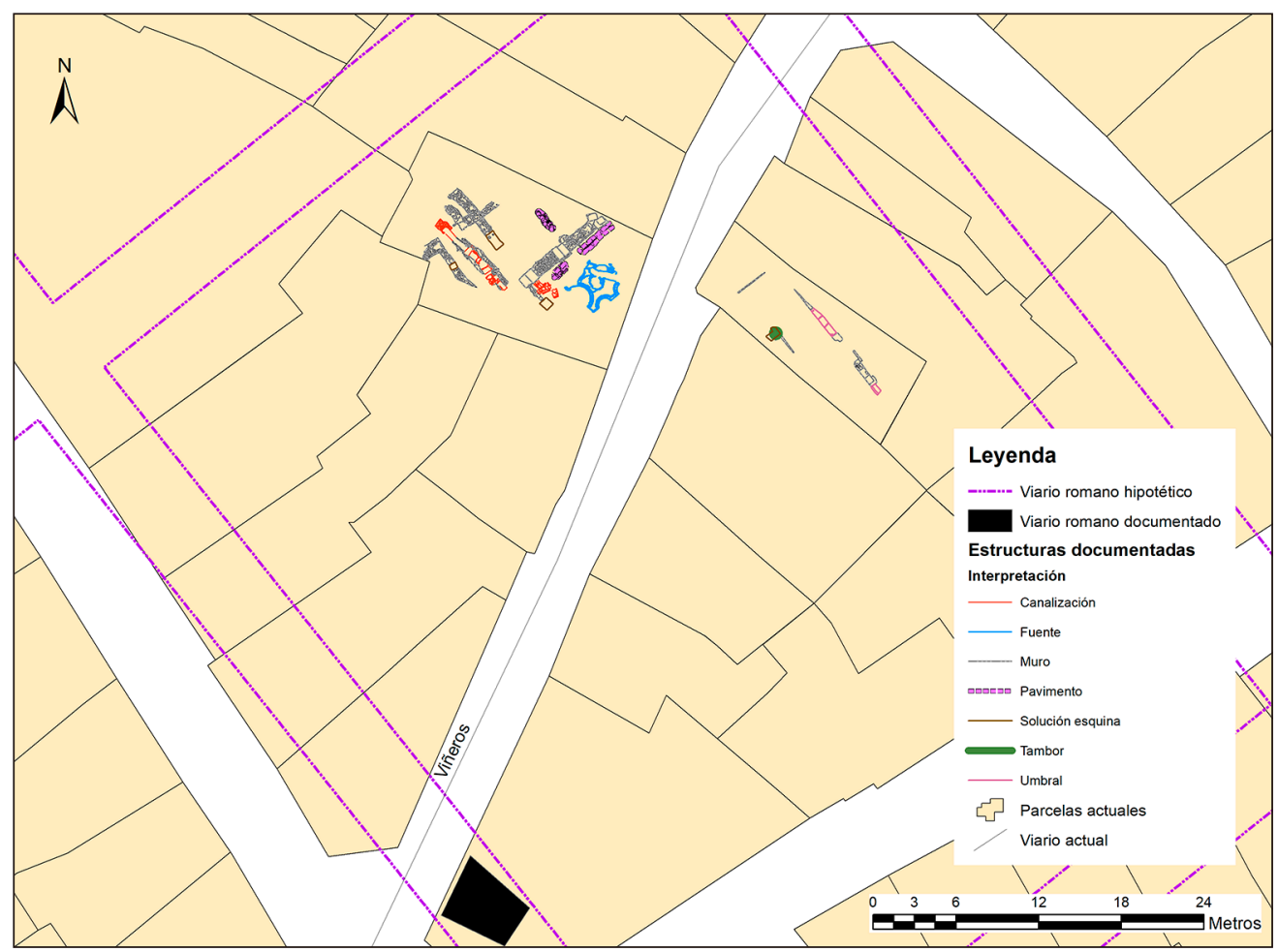

Figura 2. Planimetría que muestra la integración de datos arqueológicos en la trama urbana actual mediante el uso de SIG (fuente: elaboración del autor a partir de planimetrías del Consorcio de Mérida).

un mismo período para las estructuras documentadas: fase tardorromana. Finalmente, el examen de los aparejos y la orientación de los restos arquitectónicos permitieron discernir que los resultados de ambas intervenciones pertenecían, en realidad, al peristilo de una única vivienda, posibilitando reconocer un espacio de distribución (fig. 2).

En el siguiente caso, el concurso de los SIG posibilitó coordinar la información de una sala social de una vivienda pavimentada con un mosaico cuyas primeras noticias fueron expuestas por M. J. de Larra (1835). Con posterioridad, en 1925 J. R. Mélida publicó un dibujo realizado por M. del Albo. El mosaico citado, de temática nilótica, fue redescubierto en el año 1944 por Serra Ràfols y trasladado, finalmente, al Museo Nacional de Arte Romano, donde se conserva. A finales de la década de los ochenta del pasado siglo xx (Mateos, 1989) se produjo una excavación en el solar que contuvo los restos de la producción musiva. A principios de la década del 2000, una nueva intervención, esta vez sobre el acerado de la vía Sagasta, registró nuevas estructuras (Sánchez, 2005: 431-454). La descripción literaria de Larra, aunada al dibujo de M. del Albo publicado por J. R. Mélida (1925) y fotografías tomadas en el Museo, permitieron restituir el diseño en planta de la 


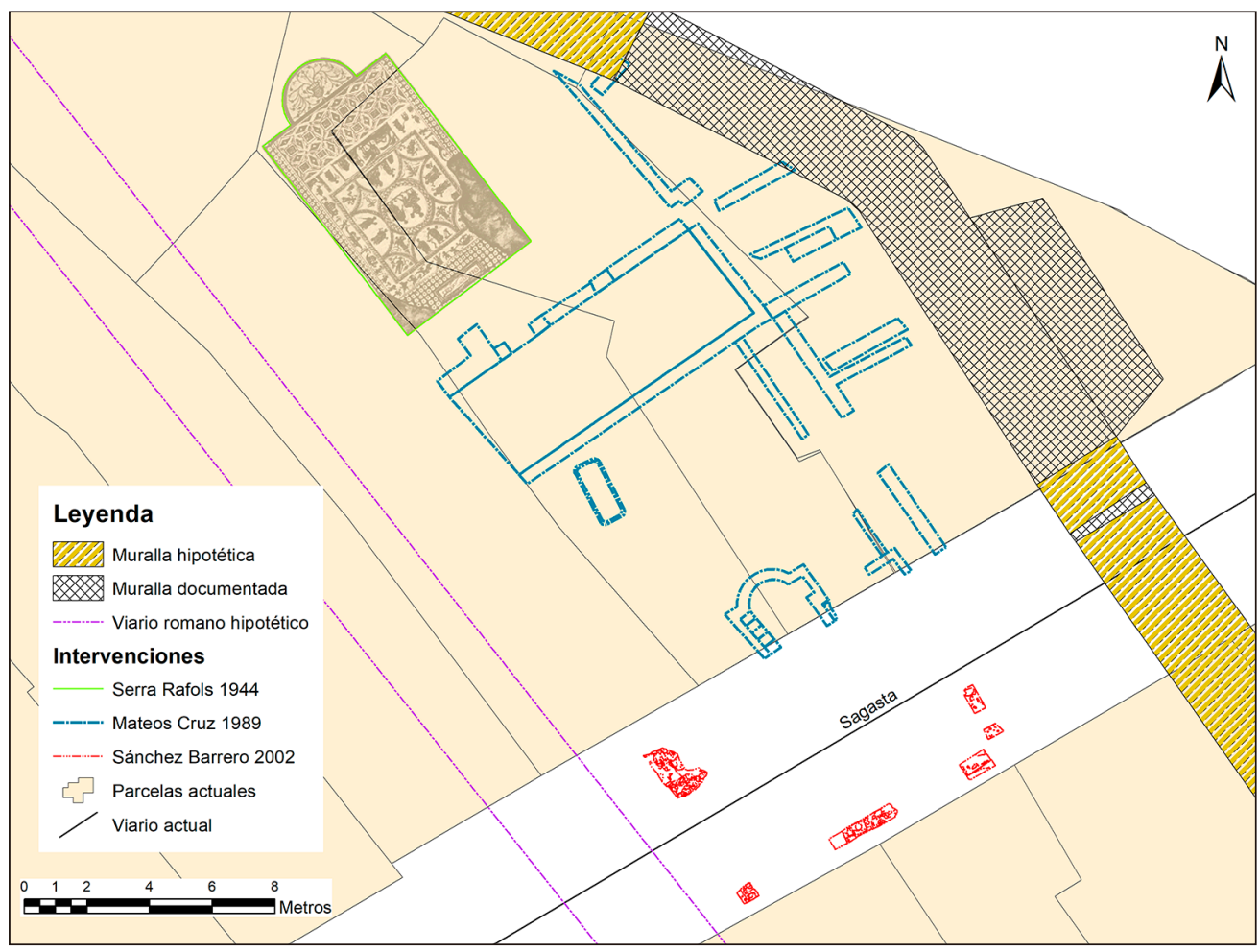

Figura 3. Planimetría que muestra la integración de documentación histórica y actual mediante el uso de SIG (fuente: elaboración del autor a partir de planimetrías del Consorcio de Mérida).

estancia e insertarla en la trama urbana de la Mérida romana, en relación con los datos de las excavaciones de los años ochenta y dos mil. El uso de los SIG facilitó el trabajo de integración de las estructuras de diferentes intervenciones realizadas a lo largo de más de un siglo, permitiendo conocer con mayor detalle la planta de la vivienda (fig. 3).

Entre las limitaciones o desventajas de esta propuesta cabe resaltar el consumo de tiempo y la dificultad de integrar datos con documentación histórica. En este sentido, el proceso de toma de datos, almacenamiento y análisis implica una gran inversión de horas que no siempre se aprecia en los resultados conseguidos. La integración con mapas históricos es compleja: si la cartografía no es trabajada con los SIG desde el principio es muy difícil obtener información significativa.

En el caso de los yacimientos urbanos, el uso de esta tecnología es fundamental para poner en relación la documentación de las distintas intervenciones arqueológicas, las cuales, como teselas de un mosaico, con el paso del tiempo, nos permitirán disfrutar de un nivel de detalle óptimo, para conocer el motivo decorativo en conjunto. 


\section{Urbanismo y edilicia doméstica emeritense: de la teoría a la práctica en algunos ejemplos}

\subsection{La trama urbana y las casas}

El objetivo de este apartado es comprobar la existencia de parcelas homogéneas y zonificación social en los diferentes sectores urbanos. La secuencia ocupacional de Augusta Emerita puede ser estudiada en tres fases: fundacional, altoimperial y tardorromana. Aunque permanecen opiniones contrarias (Canto, 2017: 341-392), la mayoría de los investigadores familiarizados con el yacimiento sostienen que Augusto fue el promotor del proyecto urbano (Mateos y Pizzo, 2018: 13-40). El trazado de la muralla y las vías intramuros generaron disfunciones en las dimensiones de las manzanas residenciales creadas en subordinación a estas construcciones. No obstante, la mayoría de los lotes debieron poseer una morfología en planta de tendencia rectangular, de diferentes tamaños, adaptándose a un trazado urbano ortogonal.

Respecto a sobre si existieron parcelas estándar en Augusta Emerita, estas solo pudieron crearse durante el urbanismo fundacional. En el estado actual de la investigación, este dato es desconocido para la totalidad del asentamiento. Nuestra hipótesis de trabajo es que el espacio intramuros contó, de manera generalizada, con manzanas residenciales en torno a unos $3.550 \mathrm{~m}^{2}$ (74 por $48 \mathrm{~m}$ ). Sin embargo, el proceso de repartición de lotes en el mundo romano siguió una pauta en la que la desigualdad sería la nota dominante, fruto de una sociedad jerarquizada. Tito Livio informa sobre suertes del doble de extensión para los equites que para los pedites en los casos de Thurii Copia o Vibo (Fentress, 2000: 18). Arqueológicamente, esta idiosincrasia ha podido ser advertida en la Cosa del siglo II a. C. En esta colonia, la gradación social se hizo patente en las manzanas residenciales más próximas al área forense, pues las viviendas de este sector poseían el doble de anchura que las casas documentadas en otras áreas urbanas (Scott, 1993: 13-63). En Augusta Emerita, la documentación arqueológica ha puesto de relieve la existencia de manzanas residenciales cuyo ancho máximo llegó a los $66 \mathrm{~m}$. Estas insulae se insertan en el sector noreste de la colonia, cerca del foro. Este fenómeno, cabría ponerlo en relación con lo registrado en Cosa; por lo que parece razonable pensar que hubo casas para dos grupos de colonos diferentes también en nuestro caso de estudio (fig. 4a). Al ser una colonia fundada por soldados veteranos, parece razonable pensar que la suerte que recibió un alto mando no fue la misma que debió recibir un militar de rango raso.

La ciudad altoimperial vive una etapa de consolidación y modificaciones significativas en el plano urbanístico. En líneas generales se asistió a diversos procesos de naturaleza opuesta que generaron tensiones entre espacios de titularidad pública y privada. De una parte, la creación del conjunto monumental de culto imperial, en época tiberiana, y la ampliación del área forense, en época flavia, se extendieron sobre cuatro y dos manzanas residenciales, respectivamente, afectando el cambio de uso de suelos a unos $25.000 \mathrm{~m}^{2}$ en los sectores centrales de la ciudad (Mateos, 2006: 321; Ayerbe et al., 2009: 816). De otra 
parte, algunas viviendas incrementaron su superficie útil a costa de espacios de titularidad pública como el intervallum de la muralla, ya desde los primeros decenios de vida de la colonia, o bien a raíz de las margines del viario, con un creciente incremento en el siglo II d. C. El impacto de estas transformaciones es lógicamente desigual en la trama urbana, saldándose a favor de los espacios públicos. Afortunadamente, la documentación arqueológica disponible para el área periurbana ha permitido plantear la existencia de un poblamiento estable extramuros, permitiendo el crecimiento de suelo destinado a las viviendas.

La ciudad vivió el impulso definitivo con la designación como sede de la Diocesis Hispaniarum durante la fase tardorromana. Esta decisión permitió sobrevivir a los principales edificios de representación de la ciudad, a la vez que la ayudó a convertirse en un centro de atracción de población, constatado a nivel arqueológico con la multiplicación de restos domésticos. A su vez, la ciudad albergó a las principales figuras de representación del poder como el vicarius y el comes hispaniarum, por lo que las viviendas debieron acomodarse a estas dignidades.

Respecto a la segunda cuestión, el debate gira en torno a si existieron zonificaciones sociales en las ciudades romanas. Los investigadores familiarizados con los yacimientos italianos han sostenido que en Roma y otros asentamientos como Ostia, Pompeya o Herculano conviven paralelamente las domus junto a apartamentos y las denominadas shop-houses (Boëthius, 1934: 159; Packer, 1967: 80-95; Wallace-Hadrill, 1994: 64-117, 2011 : 199-285). Lógicamente, la diferente respuesta constructiva está relacionada con la estratificación de la sociedad, bien atestiguada en estos yacimientos y en la literatura imperial, especialmente en la obra de Aelius Aristides (Or. 26,39 y 26,59). En el caso de Augusta Emerita, la documentación arqueológica disponible no ha registrado hasta el momento construcciones de apartamentos, dado que no se han conservado estancias en altura. ¿Quiere decir que no se construyó esta tipología? A tenor de los datos disponibles hasta el momento, la respuesta es clara. Sin embargo, cabría preguntarse si fue la Mérida romana una ciudad tan populosa como los citados asentamientos para necesitar de este tipo de estándar constructivo. Por el momento la traslación del modelo shop-house tampoco parece trasladarse a nuestra colonia, pues los inmuebles asociados a este tipo son periurbanos (Sastre y Mañas, 2014: 1107-1110).

Por otra parte, el análisis metrológico de las manzanas residenciales permite comparar la superficie teórica conocida, mediante cálculos geométricos precisos realizados con los SIG, con el área en metros cuadrados ocupados por las casas documentadas. Este trabajo solo pudo realizarse para las viviendas situadas intramuros, puesto que el espacio suburbano no cuenta con una delimitación por manzanas residenciales. Este análisis mostró un grado de conocimiento deficitario para la mayoría de la ciudad. De hecho, solo tres manzanas residenciales se conocen con un nivel de detalle (más del 80 \%) que permita extraer consideraciones significativas. Todas ellas se integran en el área de Morerías y son contiguas entre sí, con orientación noroeste-sureste: las manzanas III, 20; III, 15 y III, 10 (fig. 4b).

La lógica social apreciable en estas manzanas residenciales puede servir para conocer las pautas generales de la ciudad. En el caso de la insula III, 20, que presentó unas 

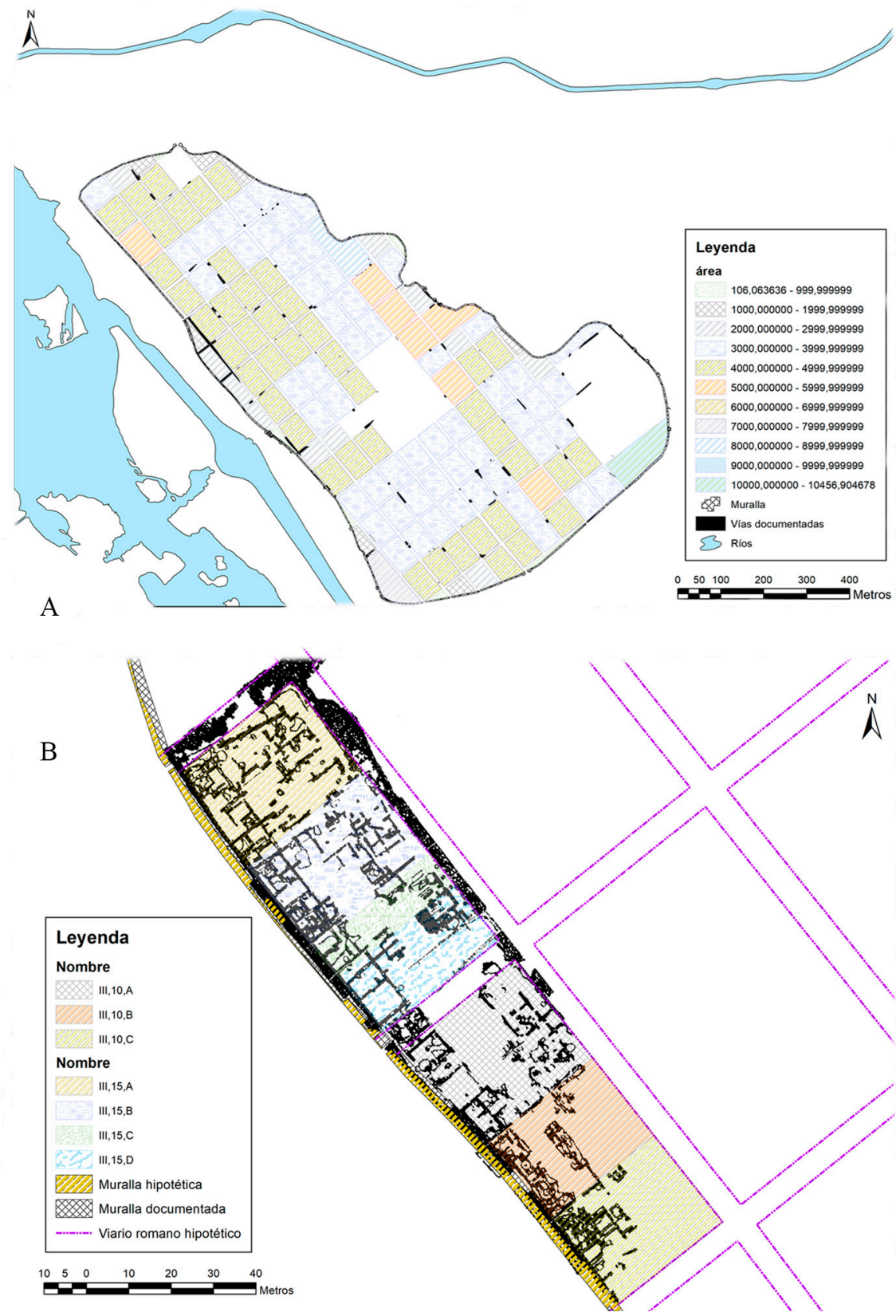

Figura 4. Mapa que muestra la desigual parcelación de manzanas residenciales (fuente: elaboración del autor a partir de planimetrías del Consorcio de Mérida).); 4b. Mapa que muestra la asimétrica distribución de casas por insulae (fuente: elaboración del autor a partir de planimetrías del Consorcio de Mérida). 
dimensiones de 78,3 x 33,1 (2.591,7 $\left.\mathrm{m}^{2}\right)$, una sola vivienda se documentó en su interior. La casa III, 20 ocupa una extensión de $1.057,4 \mathrm{~m}^{2}$, es decir, el 80,9\% de la totalidad de la manzana. La insula III, 15 contó con unas dimensiones de 78,2 x 35, $1\left(2.744,8 \mathrm{~m}^{2}\right)$. La ocupación de la manzana se debió a cuatro casas diferentes (III, 15, A; III, 15, B; III, 15, C y III, 15, D), con la siguiente parcelación: la vivienda A contó con 949,7 m²; la casa B poseía $893,7 \mathrm{~m}^{2}$; la casa C contó con $307,2 \mathrm{~m}^{2}$ y la casa D poseía $594,2 \mathrm{~m}^{2}$. Tras estos números cabe apreciar una repartición asimétrica en época tardorromana de la extensión de la manzana residencial, en la que la vivienda A ocupó el 34,6 \%, la vivienda B representó el 32,6\%, la vivienda C apenas supone el 11,2\% del total y la vivienda D representa el $21,6 \%$. Finalmente, la manzana residencial III, 10 tuvo unas dimensiones de 79,1 x 31,9 $\left(2.523,3 \mathrm{~m}^{2}\right)$. La ocupación de esta insula se debió a tres casas diferentes (III, 10, A; III, 10, B y III, 10, C), con la siguiente división de lotes: la vivienda A contó con 984,7 m²; la casa B poseía $730,8 \mathrm{~m}^{2}$ y la casa C contó con $807,8 \mathrm{~m}^{2}$. Tras estos números cabe apreciar una repartición asimétrica en época tardorromana de la extensión de la manzana residencial, en la que la vivienda A ocupó el 39\%, la vivienda B representó el 29 \% y la vivienda C supone el $32 \%$ del total. En el caso de esta última manzana, es posible apreciar que las casas esquineras ocupan mayor superficie que la casa central.

\subsection{Modelos arquitectónicos}

El marco de referencia para este tipo de estudios se fija en cuatro aspectos: geográfico, técnico, político y social. El primer factor, de carácter espacial, advierte que la posibilidad de desarrollo de una tipología arquitectónica residencial está condicionada por la extensión de la parcela urbana que se inserta, así como por la regularidad o irregularidad de la morfología del predio urbano. El segundo elemento, de naturaleza edilicia, se refiere a las condiciones para la construcción de una casa, desde las infraestructuras hasta las técnicas constructivas. El tercer parámetro, de carácter político y cultural, se basa en la conciencia de las élites dirigentes del vínculo entre status y valor simbólico de la casa. Este último aspecto facilita la transformación continua de los ambientes. Desde el punto de vista social, los promotores privados con mayores posibilidades económicas, sean colonos itálicos o locales, son los encargados de adoptar los modelos arquitectónicos (Gros, 2001: 148-150).

En el caso emeritense, el muestreo de viviendas presentado e identificado en este apartado y válido solo para tipologías domésticas de alto nivel de vida, aquellas que permitieron conocer con suficiente nivel de detalle algunos de los tres elementos que influyen en el diseño (ingreso, espacio de distribución y sala social), arrojó 37 casas.

\subsubsection{El vestíbulo}

Aulus Gellius puso de relieve que el vestibulum es un espacio vivo cuya concepción adquiere diferentes matices con el paso del tiempo. Así, en el período republicano se corresponde 
con un espacio exterior, sin contenido arquitectónico. Sin embargo, en el momento en que escribe este autor, siglo II d. C., el vestibulum se corresponde ya con un ambiente plenamente integrado en la vivienda (Lafon, 1995: 420).

Las viviendas de alto nivel de vida solían poseer varios accesos. Frecuentemente, la entrada principal de la casa solía estar reservada para el acceso de la familia y los clientes. Este ambiente tiende a conectar con el espacio de distribución del inmueble. En el caso de contar con una segunda entrada, esta apenas constituía una abertura en la fachada lateral y comunicaba con las dependencias de servicio, siendo destinada a los siervos.

Cuatro viviendas han conservado un ingreso principal con suficiente nivel de detalle para ofrecer una comparativa entre estas piezas (fig. 5). Como norma general, en época imperial el vestíbulo está asimilado ya como una pieza interna de la casa, flanqueado por otras estancias, generalmente tabernae, abiertas a la calle principal para obtener un rédito económico. Arquitectónicamente está vacío de contenido, es decir, se corresponde con un espacio diáfano tras pasar la puerta de ingreso, con diseños de tendencia cuadrada o rectangular. A pesar de ser el primer espacio que las personas ajenas a la familia conocían de la vivienda, en el caso emeritense no se realizaron grandes inversiones con la finalidad de impresionar a las visitas. De hecho, lo más destacado es la decoración pictórica

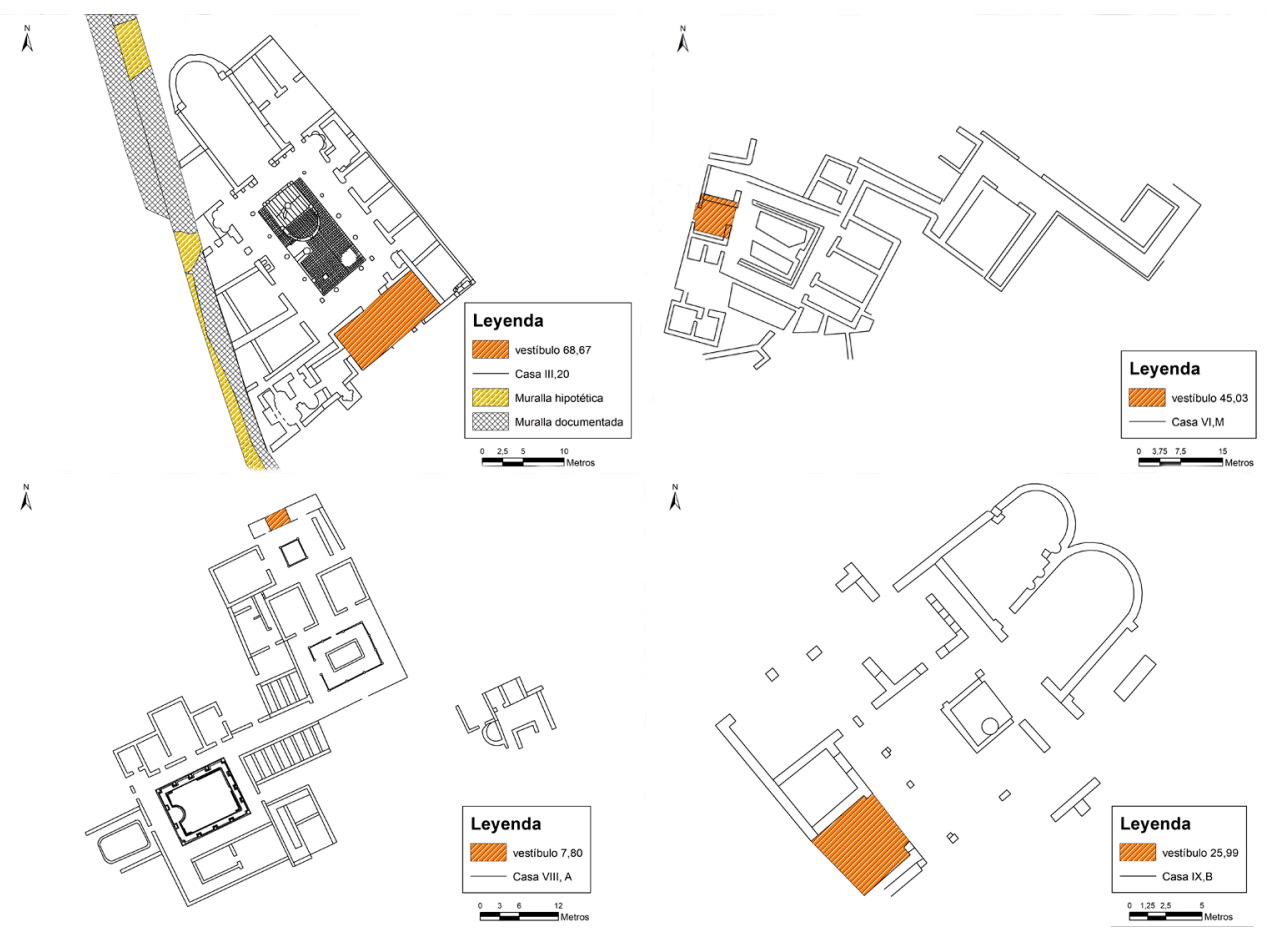

Figura 5. Tipología de plantas de vestíbulos en las viviendas de Augusta Emerita (fuente: autor). 
parietal de la Casa del Teatro, de manera sobria. En la historiografía general sobre viviendas romanas estos espacios fueron interpretados como antesalas de espera, algunas de las cuales incorporaron bancos corridos, como en la Casa del Moralista de Pompeya, para hacer más fácil la espera. Sin embargo, en el caso emeritense, ninguna vivienda presentó este elemento.

\subsubsection{Espacio de distribución descubierto}

El segundo de los factores explicativos, el espacio de distribución, se halla presente en la mayoría de los trabajos recientes sobre arquitectura doméstica hispana (Correia, 2013; Cortés, 2014: 59-93; Uribe, 2015; Corrales, 2016). Así, las tipologías se basan en el espacio de circulación que organiza la casa. De manera general, estos ambientes suelen ocupar una posición central en el diseño en planta en su concepción original, si bien transformaciones posteriores en el inmueble pueden acabar relegando este espacio a una posición excéntrica o llevar a una duplicidad si se fusionan varias viviendas en una nueva residencia. Por tanto, el análisis de esta pieza es vital para el conocimiento de una casa.

Todas las viviendas emeritenses de alto estándar contaron con un espacio de distribución abierto, normalmente de carácter central. Sin embargo, la materialización arquitectónica de este elemento poseyó variantes. El modelo más canónico, de tradición itálica, es el esquema distribuidor de atrio, originado en edad tardoarcaica (Carafa, 1998: 35-44). El tratado vitruviano consideraba cinco tipos, atendiendo a la ausencia y/o presencia de columnas; asimismo consideraba una cuestión vital la captación del agua de lluvia mediante el binomio abertura-cisterna (Vitr. De arch. 3, I-2). Este sistema de organización entra en crisis hacia el cambio de era; así, solo algunas viviendas se organizaron en torno a este espacio en época imperial en Hispania (Beltrán, 2003: 48). Por ello, no es de extrañar que este modelo resulte minoritario (28 \%) en Augusta Emerita, fundada en el año 25 a. C. (fig. 6).

En estos momentos, el diseño de vivienda organizado en torno al peristilo representa la tendencia en boga en el contexto regional hispano (Beltrán, 2003: 50). En el caso emeritense, el modelo mayoritario coincide igualmente con el peristilo (68\%). El marco de referencia de la introducción del peristilo en la arquitectura romana cabe apreciarlo en términos de la importación desde el mundo griego de un producto cultural. La demanda del mismo procedería de grupos sociales económicamente favorecidos con intenciones de diferenciarse del resto de la población (Gros, 2001: 45). Este espacio, al igual que el atrio, ve condicionada su extensión al tamaño de la parcela, por lo que existen diversos rangos de escala a tener en cuenta. En líneas generales, las viviendas ubicadas intramuros debieron ajustarse al uso de una sola pieza arquitectónica de un tamaño no superior a los $150 \mathrm{~m}^{2}$ (vivienda IV, 10: Casa de la Huerta de Otero). Por el contrario, las viviendas extramuros, con una mayor flexibilidad de parcelas, pudieron incluir una pieza de mayor dimensión o, incluso, una duplicidad de espacios que vertebraran sectores diversos de las casas (fig. 7). 
^

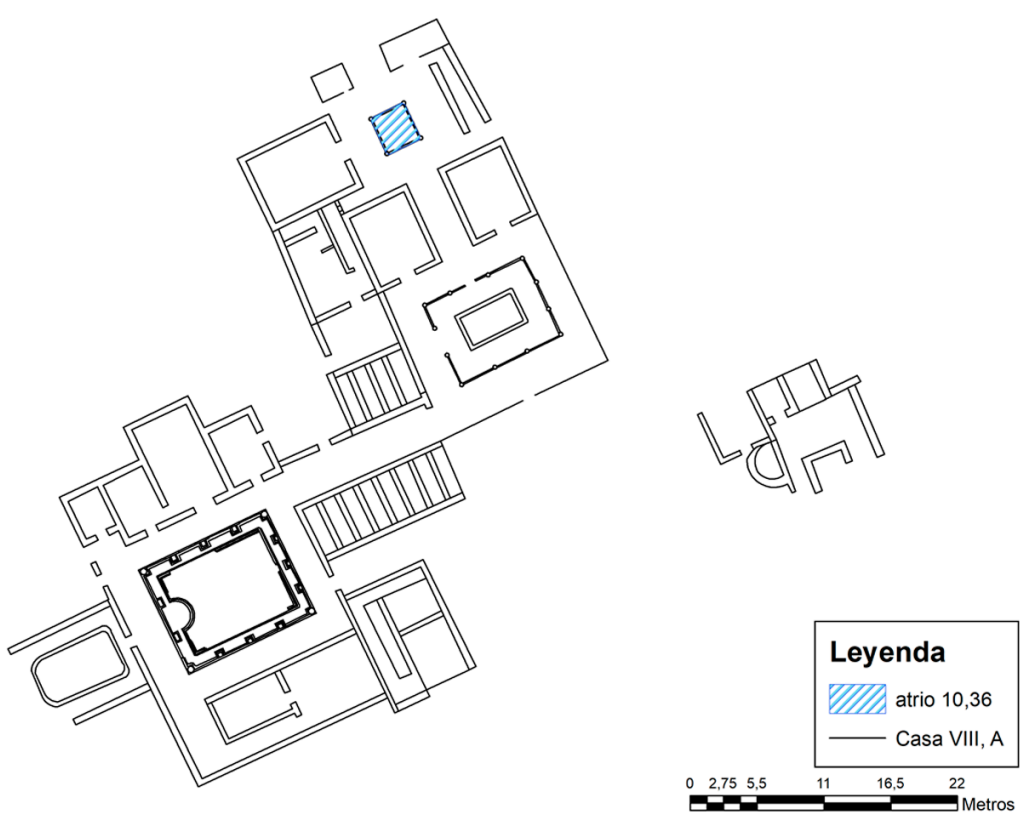

$\stackrel{N}{N}$

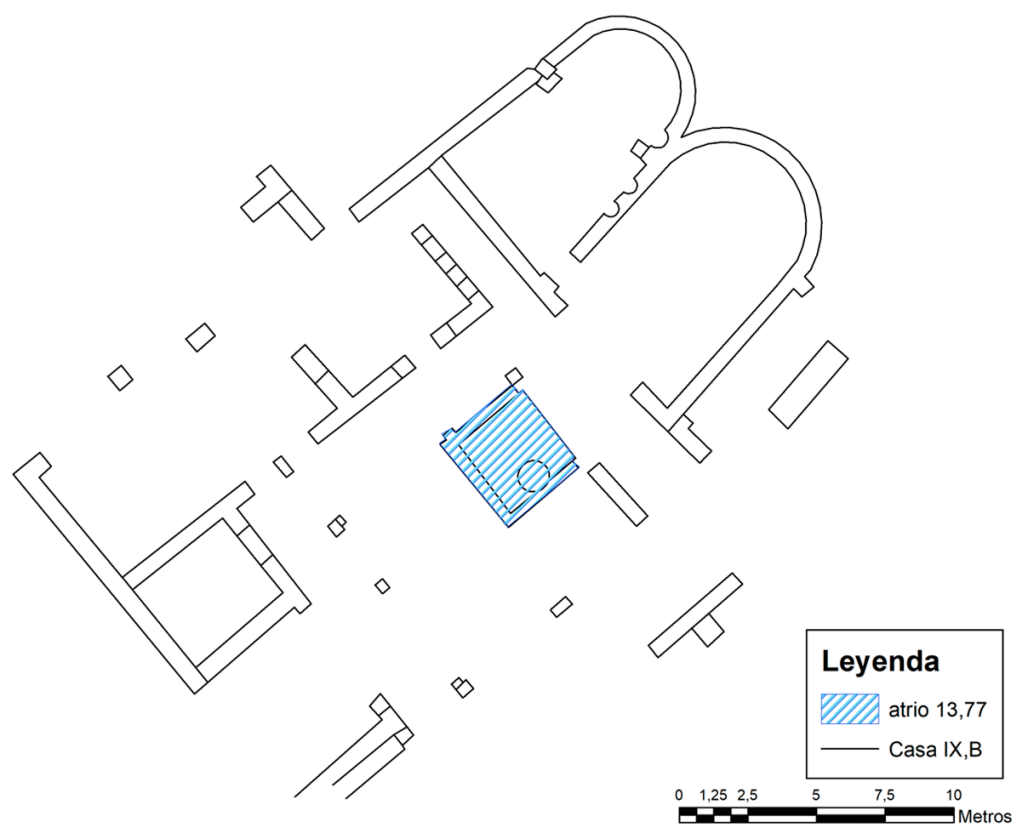

Figura 6. Tipología de plantas de atrios en las casas emeritenses (fuente: autor). 
$\AA$

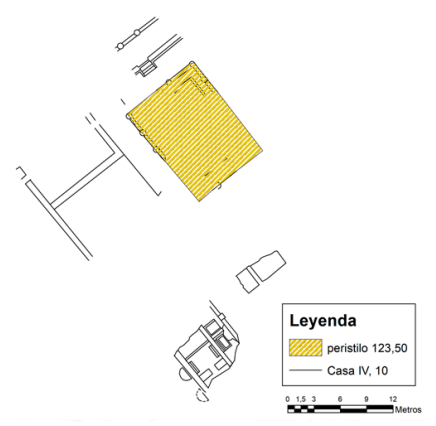

$i$

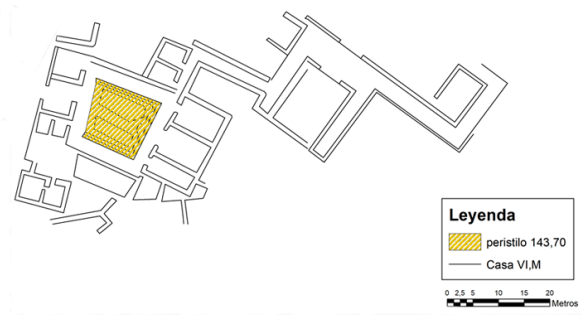

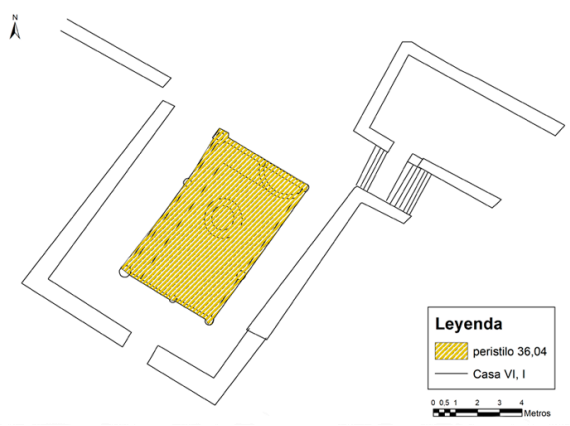

A
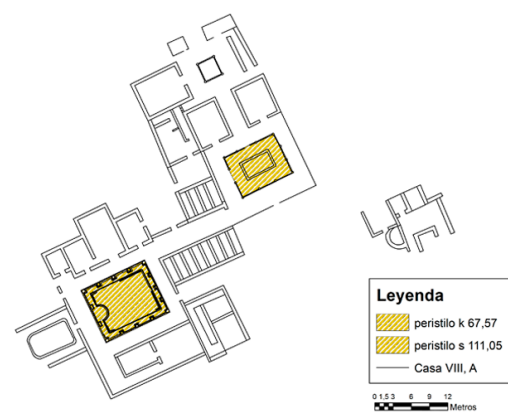

Figura 7. Tipología de plantas de peristilos en las viviendas de Augusta Emerita (fuente: autor).

En algunas ocasiones, las viviendas erigidas con una organización central en torno a un atrio decidieron añadir un peristilo en un momento posterior. La documentación hispana registró este cambio en Emporion, en la denominada Casa de las Inscripciones, la Casa del Horno o la Casa 2B, y la misma idea manifiesta la denominada Casa de Hércules de Celsa (Beltrán, 2003: 29). En todos estos casos, el peristilo se añadió como pieza independiente, al margen del atrio existente. Sin embargo, en Augusta Emerita, la denominada Casa del Teatro transformó su atrio inicial en una suerte de peristilo. Este cambio supuso la modificación sustancial del espacio de distribución, proponiendo la ampliación del espacio abierto y colocando elementos de sustentación en cada esquina. Parece razonable pensar que esta respuesta constructiva fue la mejor solución para seguir la nueva moda en formas arquitectónicas.

Asimismo, el registro arqueológico emeritense posibilitó la documentación de un último modelo de diseño de la vivienda. La variante se corresponde con una fórmula mixta en la que la organización de atrio y peristilo se conciben de manera conjunta, en un proyecto arquitectónico único. Solo una vivienda responde a este esquema organizativo $(4 \%)$, la denominada Casa del Mitreo. El único acceso conocido hasta la fecha en el inmueble abría a un atrio tetrástilo que organizaba el sector norte de la casa, en el que se localizaba el tablinum pavimentado con el mosaico cósmico. Desde el propio 
atrio, mediante un corredor, se accedía a un primer peristilo con estanque central cuyo intercolumnio estuvo decorado con pinturas de rejas de jardín. Desde el corredor sur del peristilo, mediante otro corredor, se alcanzaba el peristilo con jardín que vertebra los espacios sudoccidentales. Este modelo de vivienda necesita una gran extensión para su desarrollo. La vivienda emeritense es de carácter suburbano, situada en las inmediaciones de la muralla en su trazado sur. Al exterior de la muralla, si bien queda probado que existe una ordenación urbana siguiendo el recorrido de las vías, no es menos cierto que hubo una mayor flexibilidad en la adquisición de terrenos, al no estar sujetas las parcelas a un diseño cerrado delimitado por las vías que circundan las manzanas residenciales intramuros. Este requisito implica que solo la élite más pudiente pudiera adquirir este modelo de vivienda. En Hispania, esta tipología de viviendas que conciben esta fórmula de manera unitaria e incorporan simultáneamente un atrio y un gran peristilo es escasamente conocida. Así, apenas se han documentado otros cuatro ejemplares: en Baetulo, la domus de los Delfines y la domus de la Hiedra (Cortés, 2014: 77); en Arucci, la denominada Casa de Peristilo (Campos et al., 2018: 47), y en Bracara Augusta, la domus das Carvalheiras (Magalhães, 2013: 22).

En última instancia, conviene destacar la influencia que ejerció este modelo urbano de atrio y peristilo sobre algunas villae tardorromanas que incorporaron este diseño en su organización. En el caso de la provincia lusitana contamos con el ejemplo de la villa de El Pomar (fig. 8a), en la ciudad de Seria Fama Iulia, que estuvo dotada de un atrio tetrástilo con impluvio, el cual, a su vez, daba acceso a uno de los corredores del peristilo ajardinado (Álvarez et al., 1992: 49-71). En la provincia bética (fig. 8b-c), modelos con esta organización pueden apreciarse en Bruñel (Hidalgo, 2016: 375-386) o en Torrox (Rodríguez-Oliva y Beltrán, 2016: 631-648).

\subsubsection{Estancias de representación: el triclinium}

El tercer factor subraya la preeminencia de un determinado ambiente considerado como escenario de los ritos sociales y principales acontecimientos de vida pública en la casa. Hasta tal punto son importantes, que el diseño de la casa debe verse en relación con estos ambientes y en el contexto de las actividades sociales para las que fueron creados (WallaceHadrill, 1989: 63). Debido a límites espaciales solo valoraremos los triclinia, si bien las viviendas de alto nivel contaron además con espacios como tablinum, exedra, diaeta y oecus.

En las fuentes clásicas existieron varias voces latinas que fueron empleadas para designar los espacios destinados a las comidas sociales, entre los que cabe destacar: cenatio, cenatiuncula, cenaculum, oecus y triclinium (Foss, 1994: 84-99). Las excavaciones en la vía Sacra (Carafa, 1998: 35-44) y el Palatino (Carandini, 2007: 35-54) aludían a la existencia de espacios destinados a tal fin, con la configuración romana, ya en el siglo vi a. C. (Zaccaria, 2003: 9-15). Estas comidas organizadas eran un mecanismo de cohesión en época imperial que subrayaba la integración en el marco más amplio de la sociedad romana (Dunbabin y 


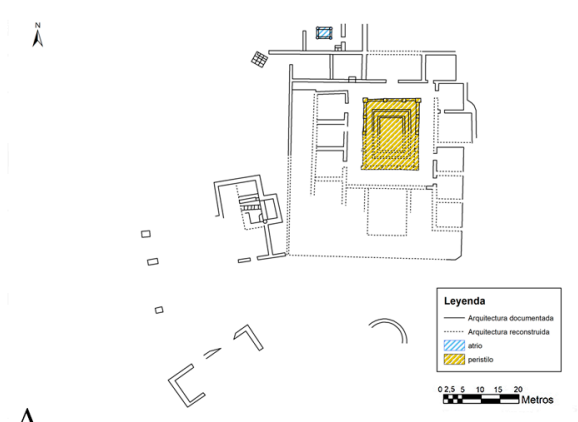

A

B
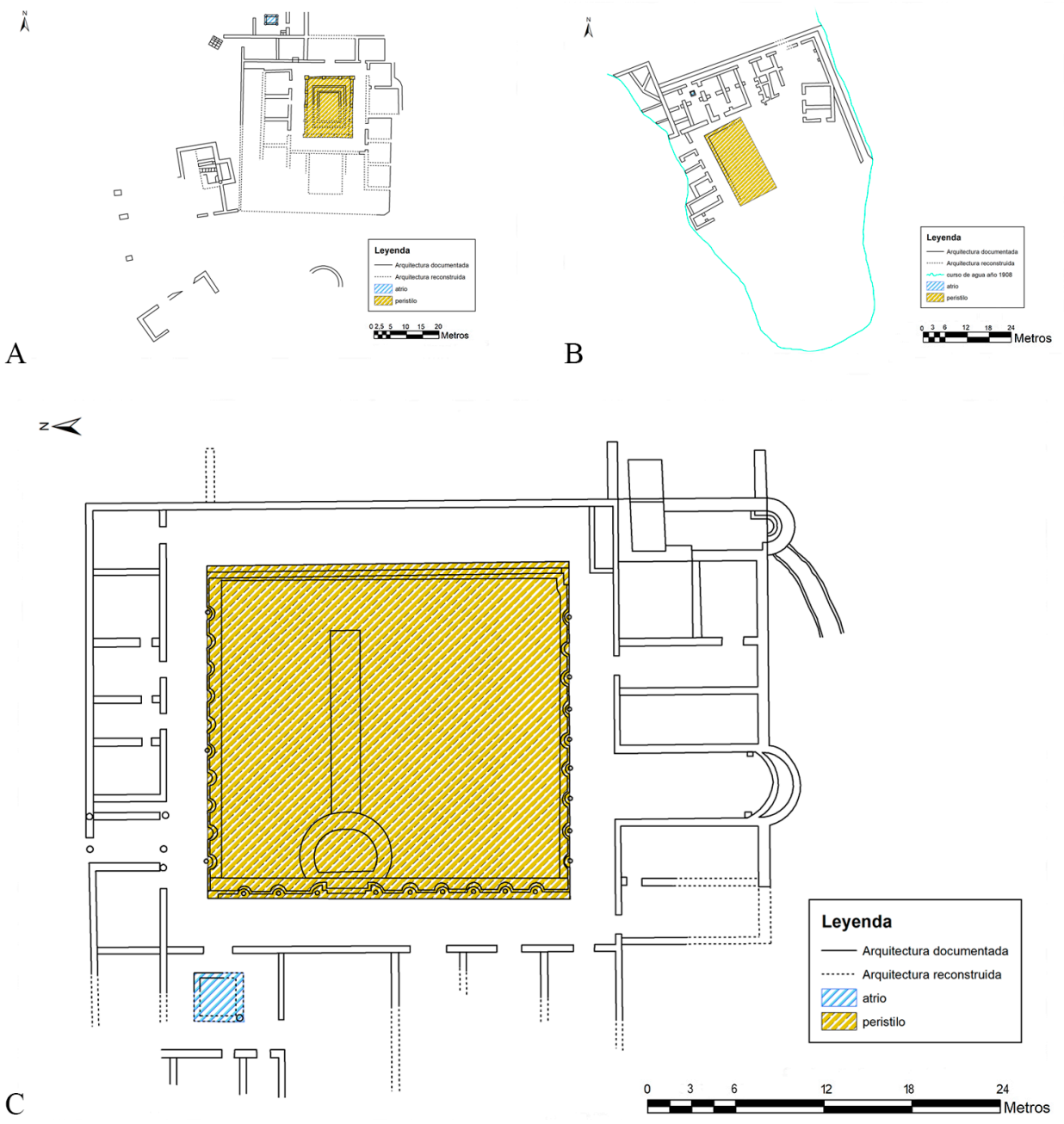

Figura 8. Planta de la villa de Bruñel (autor, a partir de Hidalgo, 2016, fig. 9); 8b. Planta de la villa del Faro de Torrox (autor, a partir de Rodríguez-Oliva y Beltrán, 2016, fig. 8); 8c. Planta de la domus de El Pomar (autor, a partir de Álvarez et al., 1992, fig. 1).

Slater, 2011 : 439). El origen del término es conocido, haciendo referencia a la pieza mueble o de obra utilizada en las comidas sociales y que poseía capacidad para tres personas. La pauta más habitual supuso una posición en planta, en eje axial con la entrada de acceso a la vivienda, orientados a un ambiente abierto, con unas dimensiones suficientes para albergar todo el mobiliario necesario para la actividad que iba a desarrollarse y una prolija decoración tanto pictórica como pavimental, de la que son características las formas "U», «T» $\mathrm{o}$ « $\mathrm{T}+\mathrm{U} »$ en mosaicos y emblemas centrales en opus sectile. Asimismo, formalmente 
i

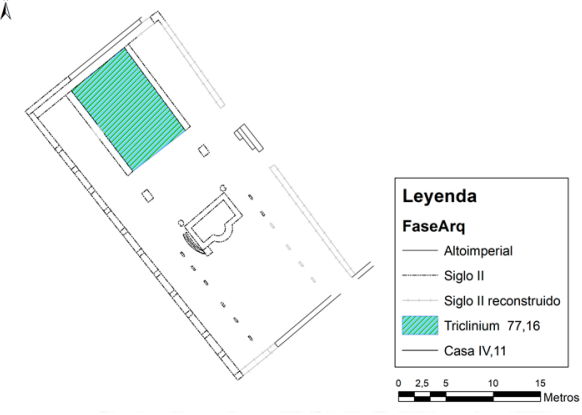

^

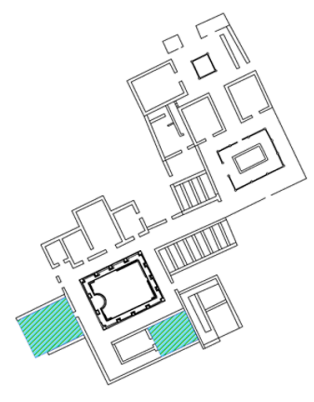

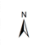
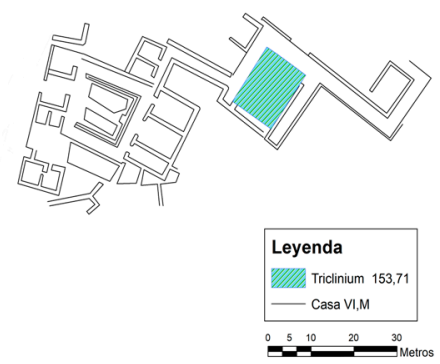

$\lambda$

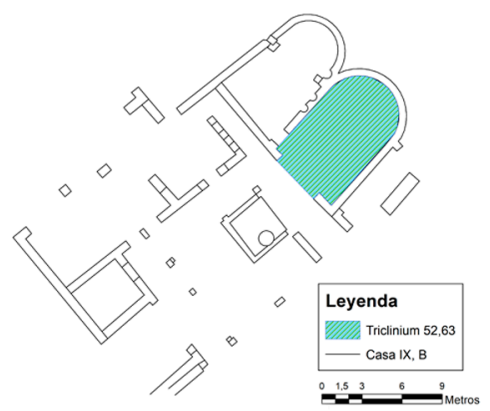

Figura 9. Tipología de plantas de salones triclinares en las casas emeritenses (fuente: autor).

suelen poseer tres accesos para facilitar el tránsito del servicio de la comida en las celebraciones (Zaccaria, 1995: 137-154).

En Augusta Emerita se han podido identificar un total de trece ambientes ${ }^{1}$. Debido a la economía de espacio no desarrollaremos aquí todos los casos documentados, sino que haremos una síntesis de los elementos comunes y su evolución en el tiempo (fig. 9a-d). Así, el primer aspecto a subrayar es la ocupación de una posición en planta preeminente, normalmente en eje con el ingreso principal de la casa o un espacio de circulación. Ello es debido a la importancia de la celebración de banquetes sociales en este tipo de viviendas de alto nivel de vida. La segunda característica de estos ambientes es la gran superficie útil que ocupan, desde el ambiente más pequeño identificado en la vivienda VIII, A (Casa del Mitreo) de $37 \mathrm{~m}^{2}$ hasta los $153 \mathrm{~m}^{2}$ del espacio examinado en la vivienda VI, M. El número de vanos de acceso también es un elemento a tener en consideración para el reconocimiento de este tipo de ambientes, documentándose los tres accesos canónicos solo en dos ejemplares (IV, 4, B: Casa de la Alcazaba; VI, M: Casa del Anfiteatro).

1. Viviendas: II, 5, A; II, 14, B; III, 7, A; IV, 4, B (Casa de la Alcazaba); VI, M (Casa del Anfiteatro); VIII, A (Casa del Mitreo: triclinium aestivum et hiemalis); II, 25; III, 15, D; III, 20 (Casa de los Mármoles); IV, 24, A; IX, A; IX, B. 
Todos los espacios identificados como triclinia estuvieron dotados de un gran aparato decorativo que engalanaba la arquitectura de la sala. Las producciones musivas fueron las elecciones predominantes en estos repertorios (8 viviendas), en los que se suelen mostrar motivos de figuras animales como pájaros (II, 14, B), ictiofauna (III, 7, A; VI, M) o escenas cinegéticas (II, 25). Solo en un caso se atestiguó una producción en sectilia pavimenta, en una producción mixta con materiales locales e importados traídos ex profeso para esta sala (IV, 4, B). Las paredes se decoraron preferentemente con técnicas pictóricas. El mayor exponente es el ejemplar documentado en la vivienda IX, B (Casa del Teatro), en el que se representan tres figuras humanas ricamente ataviadas en actitud de agasajo, siendo identificadas como integrantes de la familia propietaria dispuestos a recibir a los invitados (Mostalac, 1997: 600). Por otra parte, cabe destacar la evolución de la morfología de las estancias con el tiempo. Así, los testimonios adscritos al período altoimperial poseyeron una morfología de tendencia rectangular, mientras que las salas tardorromanas adoptaron la moda del espacio en ábside (6 viviendas), elemento que ayuda a crear un lugar central, remarcando el status de las personas. Sin embargo, la razón principal de este cambio fue la adopción del mobiliario (stibadium) en forma de sigma. Finalmente, conviene destacar uno de estos espacios por su morfología triconque (vivienda III, 15, D). Este diseño es bien conocido para complejos de escala imperial como Piazza Armerina (Carandini et al., 1982) o el palatium de Cercadilla (Hidalgo, 1998: 273-300). El modelo es importado en las viviendas urbanas de la élite dirigente, que busca con este producto diferenciarse del resto de la sociedad (Morvillez, 1993: 16).

\section{Discusión}

La transformación de un conjunto recíproco de derechos y obligaciones hacia una forma de dignidad social es fundamental para la identidad cultural romana, al menos desde la etapa de Augusto. De hecho, la sociedad ya no se expresa solo a través de una serie de acciones sociales (votar y hacer la guerra), sino también a través de un código de símbolos que define automáticamente la pertenencia al concepto de romanidad (WallaceHadrill, 2008: 452). Así, la arquitectura no es solo un contenedor estático, sino que tiene una dimensión religiosa, social y económica que genera la base para la reproducción de modelos y contiene una ideología profunda (Thébert, 1987: 400). En esta clave, el lenguaje arquitectónico doméstico provincial debe interpretarse como una referencia distintiva de diferentes identidades en la relación entre Roma y las nuevas colonias (Aiosa, 2018: 83).

La transmisión y difusión de modelos en la arquitectura doméstica urbana emeritense es el resultado de un modelo mediterráneo, de origen helenístico, que tiene como puntos de referencia generales diferentes ejemplos presentes en el contexto itálico y en las provincias occidentales. Desde el punto de vista tipológico, hay tres elementos que definen 
claramente las conexiones con los modelos mediterráneos: la entrada, el peristilo y las salas de recepción. En cuanto a los vestíbulos, Marcus Vitruvius Pollio recoge en su tratado una práctica común generalizada según la cual los espacios deben adaptarse a las necesidades sociales y a la dignidad de la representación pública: «... Quant aux dignitaires, que l'exercice des plus hautes magistratures astreint à des obligations envers leurs concitoyens, il leur faut des vestibules de style royal, des atriums élevés et des péristyles immenses..." (Vitr. De arch. 6.5.2). El escaso grado de conservación de las piezas emeritenses no ha posibilitado una comparación con la complejidad arquitectónica documentada en el área vesubiana o en el norte de África.

En la historia del hábitat mediterráneo, la evolución de la casa a pastás (mediados del siglo vir a. C.) a la casa de peristilo (v a. C.), variante con un mayor número de columnas (Pesando, 1987: 149; Lippolis et al., 2007: 278) y centro de distribución de los diferentes ambientes, representa una innovación decisiva. Esta nueva fórmula se adoptó en las regiones del Mediterráneo occidental ya en el mundo púnico, como lo demuestra la Casa de las Columnas de Kerkouane (Kélibia, Túnez), abandonada en el III a. C. (Thébert, 1987: 313). La misma forma de organizar el espacio se utilizó en dos niveles en la Casa de Peristilo 1 de Monte Iato (Palermo, Italia). El edificio tenía columnas de estilo dórico para la planta baja y columnas de estilo jónico para el primer piso, si bien la cronología de esta última no se ha podido precisar con anterioridad o posterioridad al 300 a. C. (Aiosa, 2017: 233-248).

El peristilo facilitó la organización de forma radial (mayoritariamente) o axial (minoritariamente) al guiar visualmente a los visitantes desde las fauces a las salas de recepción. Entre los años 80-79 a. C., la edilicia de la península itálica experimentó la preponderancia definitiva del peristilo con respecto al atrio. Este cambio en la configuración espacial de la casa se aleja de los modelos existentes durante la República (Fernández, 1999: 69) y es, evidentemente, el resultado de una nueva mentalidad, que tendrá una difusión generalizada en todo el mundo romano. La documentación arqueológica itálica presenta, de hecho, en diferentes situaciones geográficas, desde Campania (Adam, 2012; Dessales, 2014: 23-34; Anderson y Robinson, 2018; Dardenay, 2018: 41-51; Helg, 2018: 115-126) a la Cisalpina (Bonini, 2012: 45), un "patio porticado»: un área descubierta que tiene, en uno o más lados, un pórtico sostenido por columnas o, más raramente, pilares. El modelo se extendió por toda la región gala (Vipard, 2007: 227-277, 2009: 234-243; Alberti, 2009 : vol. IV: 39, 2018: 26-35; Auvertin, 2018: 215-232), con un aumento significativo en el uso del peristilo en la época romana, convirtiéndose en el modelo predominante en la mayoría de las ciudades, como la Maison 4 del siglo I d. C. (Binet, 1996: 88-92) en Samarobriva Ambianorum (Amiens, Francia) o la Maison Sud de la etapa flavia (Gaston y Vaxelaire, 2006: 92-95) en Vesontio (Besançon, Francia).

La introducción del peristilo fue sin duda una transformación importante para la arquitectura privada romana (Dickmann, 1997: 132). Los nuevos contextos asumieron las necesidades de recepción social dentro de las casas y en la arquitectura provincial cobró especial importancia el triclinium, predominando sobre exedras y oecus. 


\section{Consideraciones finales}

Hay muchas razones que dificultan el análisis de los modelos arquitectónicos de la edilicia doméstica emeritense. Entre las más importantes destacamos el estado de conservación y la precisión de la datación de las estructuras, frecuentemente referidas de modo genérico como fundacionales, altoimperiales o tardorromanas. Esta ausencia significativa de indicadores es causada por dos fenómenos: en primer lugar, el origen de la documentación arqueológica, condicionada por las transformaciones urbanas en los períodos históricos y la reutilización continua de espacios y materiales; en segundo lugar, la falta de publicaciones monográficas sobre grandes casas excavadas durante las últimas décadas del siglo pasado que podrían ofrecer un espacio para una interpretación correcta, especialmente en el área de Morerías.

El urbanismo es uno de los agentes principales en el devenir de las viviendas intramuros, en tanto que determina las dimensiones de las manzanas residenciales. En Augusta Emerita existieron insulae de tamaño desigual debido a dos factores: el primero de ellos motivado por la irregularidad del trazado de la muralla, condicionado por la orografía del terreno; el segundo motivado por la voluntad de reservar un espacio de mayor superficie destinado a la vivienda de los grupos sociales con un papel dirigente, próximo a los edificios públicos de poder. El grado de conocimiento sobre la distribución de parcelas es muy deficitario. Nuestro cálculo se basa en la ratio de proporcionalidad entre la extensión de las manzanas residenciales y las estructuras domésticas registradas en su interior. Así, en la mayoría de los casos conocemos la ocupación de las insulae en menos del $5 \%$. Sin embargo, existen tres manzanas de dimensiones similares, bien conocidas: III, 10; III, 15 y III, 20. El análisis microespacial de dichas manzanas reveló un patrón de ocupación donde la nota dominante es la desigualdad.

La historiografía de la arquitectura residencial de Mérida en la etapa fundacional sigue siendo un campo abierto de investigación. En el estado actual de la investigación, el único elemento notable en el registro arqueológico está representado por la introducción del modelo de articulación de la casa de peristilo (III, 16). La razón que explica esta primera recepción del modelo no solo en Mérida, sino en gran parte del territorio de Hispania, es la necesidad de establecer una serie de ciudades de nueva creación en la etapa de Augusto.

La consolidación y transformación de los modelos mediterráneos es evidente en Mérida durante toda la fase altoimperial (siglos I-II d. C.). La documentación arqueológica de este período es rica en restos de peristilos ( 7 casas) y salas de recepción (8 casas). Uno de los factores a considerar es que los diferentes indicadores arquitectónicos mencionados al principio no están documentados simultáneamente dentro de la misma casa, con la excepción de la Casa del Mitreo (vivienda VIII, A).

Un dato para reflexionar es el de la ubicación de las casas emeritenses más lujosas. De hecho, la mayoría de estas viviendas no se construyen en el centro de la ciudad, sino que se encuentran en áreas periféricas, cerca de la muralla, tanto al interior como al exterior. Este fenómeno también tiene una explicación cronológica. Durante la época fundacional es el momento en que se asigna y ocupa la mayor parte del espacio destinado a las casas. Este tipo de gestión necesaria para la distribución parcelaria dentro de la colonia deja pocas áreas 
libres para la construcción en períodos posteriores. Los propietarios que pretendían vivir en el espacio intramuros estaban condicionados por los límites de los lotes residenciales, una imposición espacial que, probablemente, no existía en las áreas fuera de la muralla.

El impulso definitivo en la configuración del paisaje doméstico de la ciudad se produce con la elección de Augusta Emerita como sede de la Diocesis Hispaniarum (hacia el 284-305 d. C.). La casa de peristilo sigue siendo el modelo predominante (8 casas) y los espacios de recepción siguen siendo una necesidad social (13 casas). En la morfología de los espacios se abre una nueva tendencia hacia el uso de la mesa en sigma, que genera formas de ábside ( 5 casas).

La mayoría de las casas en Mérida siguen un esquema axial con el vestíbulo, el peristilo y las salas de recepción. El ejemplo de este período que mejor se adapta a este modelo de casa es el III, 20 (Casa de los Mármoles). El esquema axial de la casa se concibió ya en fase altoimperial, si bien en las distintas fases de uso la casa se vio afectada por diferentes transformaciones. Una modificación interesante es la ubicación del balneum desde el ala occidental a la fachada de la casa, ocupando parte de la vía pública, con la construcción de tres ábsides para el baño y un pequeño espacio interno, probablemente un apodyterium. La segunda transformación consistió en la introducción de un crucero con extremos de ábsides rematados cada uno con tres nichos, construidos con ladrillos reutilizables. Este elemento arquitectónico sirve para reforzar el esquema axial y ser utilizado, al mismo tiempo, como antesala para la recepción de personas que esperan ser recibidas por el dominus. Una de las casas de Mérida, I, 13, A (siglo iv d. C.), quizás tenía un espacio similar, pavimentado con un rico mosaico. La última gran transformación se planeó en fase con la construcción del crucero, en la que se incorporó el ábside al triclinium. Estos cambios se suceden durante un amplio período de tiempo, entre los siglos III-IV d. C. (Alba, 2004: 75).

En síntesis, la totalidad de las casas emeritenses de alto estándar presentaron un diseño organizado en torno a un espacio de circulación abierto, ya sea conceptualizado como patio, atrio o peristilo. Desde la fase fundacional, las plantas axiales con vestíbulo, peristilo y sala de recepción representaban la secuencia arquitectónica más exitosa en relación con las necesidades de representación social de un grupo con un estándar de vida alto. Estos proyectos de construcción continuarán predominando durante los siglos primero y segundo, sin grandes transformaciones. Las mismas opciones arquitectónicas continuarán vigentes durante la fase tardorromana (siglos III-IV d. C.), con la novedad de las salas de recepción con ábsides.

\section{Nota}

Este trabajo se encuadra en la investigación del autor "Arqueología de los espacios políticos. Arquitectura doméstica urbana romana», del Programa Estatal Juan de la Cierva Incorporación (IJC2018-037041-I), así como en el proyecto I+D+i orientado a los retos de la sociedad, "Arquitectura residencial y espacio urbano en Augusta Emerita" (PID2019105376GB-C44), IP1: Pedro Mateos Cruz, IP2: Antonio Pizzo. 


\section{Fuentes}

ARISTIDES, Discursos (Introducción, traducción y notas de F. Gascó y A. Ramírez de Verger; revisada por J. Gil), Editorial Gredos, Madrid, 1997.

VITRUVIUS, De l'architecture, Livre VI (Texte établi, traduit et commenté par Louis Callebat), Collection des Universités de France, Les Belles Lettres, París, 2004.

\section{Bibliografía}

ADAM, J. P., 2012, La maison romaine, H. Clair, Arlés.

AIOSA, S., 2017, La Casa a Peristilio 1 di Monte Iato: considerazioni per una nuova ipotesi ricostruttiva, en L. M. CALIÒ y J. DES COURTILS (eds.), L'architettura greca in Occidente nel III secolo a.C., Thiasos Monografie 8, "Sapienza» Università di Roma, Roma, 233-248.

AIOSA, S., 2018, Architettura domestica e identità. Riflessioni sul quartiere ellenisticoromano, en V. CAMINNECI, M. C. PARELLO, M. S. RIZZO y C. SORACI (eds.), Agrigento ellenisticoromana. Coscienza identitaria e margini di autonomia, Edipuglia, Bari, 83-102.

ALBA, M., 2004, Arquitectura doméstica, en X. DUPRÉ (ed.), Colonia Augusta Emerita, L'Erma di Bretschneider, Roma, 67-85.

ALBERTI, G., 2009, La maison urbaine galloromaine en Gaule Belgique et dans les Germanies, Tesis doctoral inédita, Université de Strasbourg.

ALBERTI, G., 2018, La romanisation de l'habitat urbain après la conquête. L'exemple des villes de Gaule Belgique et des Germanies, Archéopages 46, 26-35.

ALLISON, P., 1992, The relationship between wall-decoration and room-type in Pompeian houses: a case study of the Casa della Caccia Antica, Journal of Roman Archaeology 5, 235-249.

ALLISON, P., 2004, Pompeian Households: An Analysis of the Material Culture, Monographs 42, University of California, Los Ángeles.
ÁlVAREZ, J., ÁLVAREZ, J. M. y RODRÍGUEZ, G., 1992, La casa romana de "El Pomar». Jerez de los Caballeros (Badajoz), Museo Nacional de Arte Romano, Mérida.

ÁLVAREZ, J. M., 1997, La influencia africana en el mosaico hispanorromano: algunas consideraciones, Anas 10, 39-50.

ANDERSON, M. A. y ROBINSON, D., 2018, House of the Surgeon, Pompeii. Excavations in the Casa del Chirurgo (VI 1, 9-10.23), Oxbow, Oxford.

AUVERTIN, R., 2018, Habiter dans les agglomérations du nord de la Gaule (I-IIIè siècle) : Analyse architecturale de la maison romaine, Tesis doctoral inédita, Lille 2.

AYERBE, R., BARRIENTOS, T. y PALMA, F., 2009, Los complejos forenses de Augusta Emerita, en R. AYERBE, T. BARRIENTOS y F. PALMA (eds.), El Foro de Avgvsta Emerita. Génesis y evolución de sus recintos monumentales, Anejos de Archivo Español de Arqueología LIII, CSIC, Mérida, 667-832.

BELTRÁN, M., 2003, La casa hispanorromana. Modelos, Bolskan 20, 13-63.

BERMEJO, J., 2014, Arqueología de los espacios domésticos romanos: condiciones de vida y sociedad en la Meseta nordeste durante el período imperial, Excma. Diputación Provincial de Soria, Soria.

BINET, E., 1996, Le site antique du «Palais des Sports " à Amiens, Revue du Nord 318, 83-96.

BOËTHIUS, A., 1934, Remarks on the development of domestic architecture in Rome, American Journal of Archaeology 38, 158-170. 
BONINI, P., 2006, La casa nella Grecia romana. Forme e funzioni dello spazio privato fra i e vi secolo, Quasar, Roma.

BONINI, P., 2012, Aree scoperte, en F. GHEDINI y M. ANNIBALETTO (eds.), Atria longa patescunt. Le forme dell'abitare nella Cisalpina romana, Quasar, Roma, 45-69.

CAMPOS, J. M., BERMEJO, J., ARÉVALO, A., BAENA, E., BERMEJO, A., CORRALES, A., DE PAZ, M., FERNÁNDEZ, L., GARRIGUET, J. A., GONZÁLEZ, J., LEÓN-MUÑOZ, A., MARFIL, F., MORENO, E., NEIRA, L., PANZRAM, S., RUIZ, J. M., RUIZ, A., RUIZ, A., SÁNCHEZ, L. J., TOSCANO-PÉREZ, C., VAQUERIZO, D. y VIDAL-TERUEL, N. O., 2018, El mundo urbano de la Bética: breve síntesis de las ciudades de los Conventus Hispalensis y Astigitanus. Ciudades del Conventus Hispalensis, en J. M. CAMPOS y J. BERMEJO (eds.), Ciudades romanas de la provincia Baetica, Universidad de Huelva, Huelva, 29-202.

CANTO, A., 2017, Treinta años de reflexiones sobre la Emerita cesariana: De lo que Dión Casio nunca dijo, a la alineación astronómica de la ciudad y los auspicia de César, en T. NOGALES (ed.), Lusitania romana: del pasado al presente de la investigación, Museo Nacional de Arte Romano de Mérida, Mérida, 341-392.

CARAFA, P., 1998, Le domus tardo arcaiche della Sacra via e l'origine della casa italica ad atrio, en M. PEARCE y M. TOSI (eds.), Papers from the EAA Third Annual Meeting at Ravenna 1997. Vol. II: Classical and Medieval, BAR. International Series 718, Archaeopress, Oxford, 35-44.

CARANDINI, A., 2007, Roma. Il primo giorno, Laterza, Roma-Bari.

CARANDINI, A., RICCI, A. y DE VOS, M., 1982, Filosofiana, la villa di Piazza Armerina: immagine di un aristocratico romano al tempo di Costantino, Flaccovio, Palermo.

CHAMIZO, J. J., 2006, Nuevos datos sobre la domus situada al sur del Foro de la Colonia, Mérida, Excavaciones Arqueológicas 9, 243-260.

CORRALES, A., 2016, La arquitectura doméstica de Augusta Emerita, Anejos de Archivo Español de Arqueología LXXVI, CSIC, Madrid.
CORREIA, V., 2013, A arquitectura doméstica de Conimbriga e as estruturas económicas e sociais da cidade romana, Centro de Estudos Arqueológicos das Universidades de Coimbra e Porto, DirecçãoGeral do Património Cultural, Liga de Amigos de Conimbriga, Coímbra.

CORTÉS, A., 2014, Clasificación tipológica de la arquitectura doméstica romana. Reflexiones a partir de las ciudades del Noreste peninsular, Pyrenae 45, 59-93.

DARDENAY, A., 2018, Restituer l'espace domestique à Herculaneum grâce aux outils informatiques de reconstruction virtuelle. Enjeux et problèmes, Anabases 27, 41-51.

DESSALES, H., 2014, Du jardin aux jardinières : l'évolution des péristyles dans l'habitat romain, en P. VAN OSSEL y A. M. GUIMIER (coords.), Archéologie des jardins: analyse des espaces et méthodes d'approche, Éditions Mergoil, Montagnac, 23-34.

DICKMANN, J. A., 1997, The peristyle and the transformation of domestic space in Hellenistic Pompeii, en R. LAURENCE y A. WALLACE-HADRILL (eds.), Domestic space in the Roman world: Pompeii and beyond, Portsmouth, 121-136.

DUNBABIN, K. y SLATER, W., 2011 , Roman dining, en M. PEACHIN (ed.), The Oxford Handbook of Social Relations in the Roman World, Oxford University Press, Oxford, 438-466.

ELLIS, S., 1999, Theories of circulation in Roman houses, en A. LESLIE (ed.), Theoretical Roman Archaeology and Architecture: The Third Conference Proceedings, Cruithne Press, Glasgow, 75-98.

FENTRESS, E., 2000, Frank Brown, Cosa, and the idea of a Roman city, en E. FENTRESS (ed.), Romanization and the city. Creation, transformations and failures, Journal of Roman Archaeology 38, Portsmouth, 11-24.

FERNÁNDEZ, P. A., 1999, La casa romana, Akal, Madrid.

FOSS, P., 1994, Kitchens and dining rooms at Pompeii: the spatial and social relationship of cooking to eating in the Roman household, Tesis doctoral inédita, University of Michigan. 
GASTON, C. y VAXELAIRE, L., 2006, Domus du Palais de Justice, en C. STOULLIG (dir.), De Vesontio à Besançon, Chaman Edition, Neuchâtel, 92-95.

GINOUVÈS, R., 1998, Dictionnaire méthodique de l'architecture grecque et romaine. Tome III. Espaces architecturaux, bâtiments et ensembles, Collection de l’Ecole Française de Rome 84, Roma.

GROS, P., 2001, L'architecture romaine: $d u$ début du IIIe siècle av. J.-C. à la fin du Haut-Empire. Maisons, palais, villas et tombeaux, Picard, París.

HELG, R., 2018, Dalla domus alla strada, dalla strada alla città. Il contributo dell'edilizia privata alla fisionomia di Pompei ed Ercolano, en M. LIVADIOTTI, R. BELLI, L. M. CALIÒ y G. MARTINES (eds.), Theatroeideis. L'immagine della città, la città delle immagini, Thiasos Monografie 11/2, «Sapienza» Università di Roma, Roma, 115-126.

HIDALGO, R., 1998, El triclinium triconque del palatium de Córdoba, Anales de Arqueología Cordobesa 9, 273-300.

HIDALGO, R., 2016, La villa romana de Bruñel (Quesada, Jaén), en R. HIDALGO (ed.), Las villas romanas de la Bética, Vol. II, Universidad de Sevilla, Sevilla, 375-386.

HILLIER, B. y HANSON, J., 1984, The social logic of space, Cambridge University Press, Cambridge.

LAFON, X., 1995, Dehors ou dedans ? Le vestibulum dans les domus aristocratiques à la fin de République et au début de l’Empire, Klio 77, 405-423.

LARRA, M. J., 1835, Las antigüedades de Mérida. Primer artículo, Revista Mensajero 82, Madrid.

LARRA, M. J., 1835, Las antigüedades de Mérida. Segundo y último artículo, Revista Mensajero 91, Madrid.

LIPPOLIS, E., LIVADIOTTI, M. y ROCCO, G., 2007, Architettura greca. Storia e monumenti del mondo della polis dalle origini al $v$ secolo, Mondadori, Milán.

MAGALHÃES, F., 2013, Arquitectura doméstica em Bracara Augusta, Revista de Ciências Sociais 1, 15-32.
MATEOS, P., 1989, Informe sobre la intervención arqueológica realizada en el solar $n .^{\circ} 41-43$ de la calle Sagasta (Mérida), Informe inédito (n. ${ }^{\circ}$ de intervención 49), Departamento de Documentación del Consorcio de la Ciudad Monumental de Mérida.

MATEOS, P., 2006, El Foro Provincial de Augusta Emerita: un conjunto monumental de culto imperial, en P. MATEOS (ed.), El Foro Provincial de Augusta Emerita: un conjunto monumental de culto imperial, Anejos de Archivo Español de Arqueología XLII, CSIC, Madrid, 315-354.

MATEOS, P., 2011, Topografía y urbanismo en Augusta Emerita, en J. M. ÁLVAREZ y P. MATEOS (eds.), Actas Congreso Internacional 1910-2010. El yacimiento emeritense, Ayuntamiento de Mérida, Mérida, 127-144.

MATEOS, P. y PIZZO, A., 2018, El teatro y anfiteatro romanos de Augusta Emerita. Aspectos arqueológicos, cronológicos y urbanísticos, en P. MATEOS (ed.), La scaenae frons del teatro romano de Mérida, Anejos de Archivo Español de Arqueología LXXXVI, CSIC, Mérida, 13-40.

MÉLIDA, J. R., 1925, Catálogo Monumental de España. Provincia de Badajoz (1907-1910), Ministerio de Instrucción Pública y Bellas Artes, Madrid.

MORVILLEZ, E., 1993, Forme et évolution des salles de réception des grandes demeures tardives du Bassin méditerranéen occidental (IVe-VIe siècles), Tesis doctoral inédita, Université Paris-Sorbonne.

MOSTALAC, A., 1997, El programa pictórico de la estancia absidiada F de la Casa Basílica de Mérida, en R. TEJA y C. PÉREZ (eds.), Congreso Internacional: La Hispania de Teodosio, Junta de Castilla y León, Salamanca, 581-603.

NEVETT, L., 1997, Perceptions of domestic space in Roman Italy, en B. RAWSON y P. WEAVER (eds.), The Roman family in Italy. Status, sentiment, space, Oxford University Press, Oxford.

PACKER, J., 1967, Housing and Population in Imperial Ostia and Rome, Journal of Roman Studies 57/1, 80-95.

PALMA, F., 2005, Una posible domus, cerca del Foro de la Colonia, y su reutilización en época 
tardoantigua, Mérida. Excavaciones Arqueológicas 8, 247-260.

PEÑALVER, T., 2019, Áreas de servicio, producción y almacenaje en los espacios domésticos romanos del territorio valenciano, Pyrenae 50/2, 105-131.

PESANDO, F., 1987, Oikos e ktesis. La casa greca in età classica, Quasar, Perugia.

RIGGSBY, A., 1997, 'Public' and 'private' in Roman culture: the case of the cubiculum, Journal of Roman Archaeology 10, 36-56.

REBUFFAT, R., 1969, Maisons à péristyle d'Afrique du Nord : répertoire de plans publiés, Mélanges de l'École Française de Rome 81 (2), 659-724.

REBUFFAT, R., 1974, Maisons à péristyle d'Afrique du Nord : répertoire de plans publiés II, Mélanges de l'École Française de Rome 86 (1), 445-499.

RODRÍGUEZ-OLIVA, P. y BELTRÁN, J., 2016 , Villa romana del Faro de Torrox (Torrox, Málaga), en R. HIDALGO (ed.), Las villas romanas de la Bética, Vol. II, Universidad de Sevilla, Sevilla, 631648.

SÁNCHEZ, P. D., 2005, Trabajo desarrollado por el Equipo de Seguimiento de Obras durante el año 2002, Mérida. Excavaciones Arqueológicas 8, 431-454.

SAQUETE, J. C., 2005, Territorios y gentes en el contexto histórico de la fundación de la colonia Augusta Emerita, en T. NOGALES (ed.), Augusta Emerita. Territorios, espacios, imágenes y gentes en Lusitania romana, Museo Nacional de Arte Romano, Mérida, 175-195.

SASTRE, I. y MAÑAS, I., 2014, Casas-factoría de época fundacional en Augusta Emerita. Una vivienda dedicada a la producción de vino, en J. M. ÁLVAREZ, T. NOGALES e I. RODÀ (eds.), Centro y periferia en el mundo clásico, Museo Nacional de Arte Romano, Mérida, 1107-1110.
SCOTT, R. T., 1993, The houses, en V. J. BRUNO y R. T. SCOTT (eds.), Cosa IV, University Park, American Academy in Rome - Pennsylvania State University Press, 13-63.

THÉBERT, Y., 1987, Vida privada y arquitectura doméstica en el África romana, en P. ARIÈS y G. DUBY (eds.), Historia de la vida privada. Del Imperio romano al año mil, Taurus, Madrid, 304-401.

URIBE, P., 2015, La arquitectura doméstica urbana y romana en el valle medio del Ebro (siglos II a. C. - III d. C.), Aquitania 35, Burdeos.

VIPARD, P., 2007, Maison à péristyle et élites urbaines en Gaule sous l'Empire, Gallia 64, 227-277.

VIPARD, P., 2009, L'habitat urbain en Gaule sous le Principat, en Y. LE BOHEC (dir.), Rome et les provinces de l'Europa occidentale jusqu'à la fin du principat: conquêtes et stratégies, Ed. du Temps, Pornic, 234-243.

WALLACE-HADRILL, A., 1988, The social structure of the Roman house, Papers of the British School at Rome 56, 43-97.

WALLACE-HADRILL, A., 1989, Patronage in Roman society: from Republic to Empire, en A. WALLACE-HADRILL (ed.), Patronage in Ancient Society, Routledge, Londres-Nueva York, 63-88.

WALLACE-HADRILL, A., 1994, Houses and society in Pompeii and Herculaneum, Princeton University Press, Princeton.

WALLACE-HADRILL, A., 2008, Rome's Cultural Revolution, Cambridge University Press, Cambridge.

WALLACE-HADRILL, A., 2011 , Herculaneum. Past and future, Frances Lincoln Ltd with the Packard Humanities Institute, Londres.

ZACCARIA, A., 1995, Spazio privato e spazio pubblico nella città romana, Collection de l'École Française de Rome 210, Roma.

ZACCARIA, A., 2003, More regio vivere. Il banchetto aristocratico e la casa romana di età arcaica, Quasar, Roma. 
